\title{
Role of Krebs von den Lungen-6 (KL-6) in Assessing Hypersensitivity Pneumonitis
}

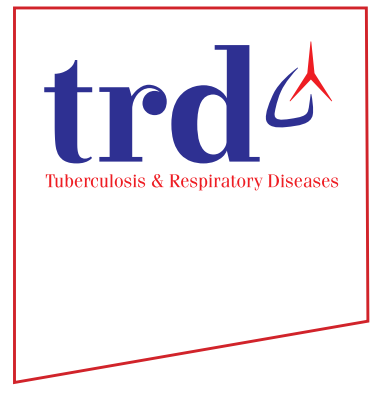

Amira Ismail Mostafa, M.D. ${ }^{1 \oplus}$, Ayman Elsayed Salem, M.D. ${ }^{1}$, Heba Allah Moussa Ahmed, M.D. ${ }^{1}$, Aml Ibrahim Bayoumi, M.D. ${ }^{1}$, Radwa M. Abdel Halim, M.D. ${ }^{2}$ and Rasha M. Abdel Samie, M.D. ${ }^{3}$ Departments of ${ }^{1}$ Chest Diseases, ${ }^{2}$ Chemical Pathology, ${ }^{3}$ Internal Medicine, Faculty of Medicine, Cairo University, Shiekh Zayed City, Egypt

Background: Hypersensitivity pneumonitis (HP) is an increasingly recognized form of diffuse parenchymal lung disease. Krebs von den Lungen-6 (KL-6) is now classified as a human MUC1 mucin protein, and regenerating type II pneumocytes are the primary cellular source of KL-6/MUC1 in the affected lungs of patients with interstitial lung diseases (ILD). Serum KL-6/MUC1 levels have been demonstrated to be useful for the evaluation of various ILD. To determine the role of circulating KL-6 in evaluating the disease activity and management of HP.

Methods: An observational cross-sectional study was conducted on 51 patients with HP and 20 healthy controls. Serum KL-6 levels were measured in both groups. Patients were further assessed based on chest high-resolution computed tomography (HRCT), pulmonary function test, 6-minute walk test, echocardiography, bronchioalveolar lavage, and/or transbronchial biopsy. Patients were divided into the fibrotic and non-fibrotic groups according to the HRCT findings.

Results: The median serum KL-6 levels were significantly higher in HP patients as compared to the control group. The median serum KL-6 levels were found to be higher in the non-fibrotic HP group $(1,900 \mathrm{IU} / \mathrm{mL})$ as compared to the fibrotic group $(1,200 \mathrm{IU} / \mathrm{mL})$. There was a significant inverse correlation between serum KL-6 serum level and the dose of steroids as well as the duration of steroid therapy.

Conclusion: The presence of higher KL-6 levels in the non-fibrotic HP group implies its enhanced production by regenerating pneumocytes in response to alveolar injury. The significant association between serum KL-6 levels and the dose and the duration of steroid therapy emphasizes the significant role of steroids in the stabilization of the disease.

Keywords: Hypersensitivity Pneumonitis; Serum Biomarker; KL-6; Assessment of Severity

Address for correspondence: Amira Ismail Mostafa, M.D.

Department of Chest Diseases, Faculty of Medicine, Cairo University, Dar Misr District 12, Shiekh Zayed City, Giza Governorate, Egypt

Phone: 20-01224977400

E-mail: dramira755@gmail.com

Received: Oct. 9, 2020

Revised: Dec. 26, 2020

Accepted: Apr. 5, 2021

Published online: Apr. 6, 2021

(c) It is identical to the Creative Commons Attribution Non-Commercial License (http://creativecommons.org/licenses/by-nc/4.0/).

\section{Introduction}

Hypersensitivity pneumonitis (HP) formerly recognized as extrinsic allergic alveolitis is the consequence of immunologically mediated type III and type IV hypersensitivity reaction caused by recurrent exposure to various inhaled antigenic stimuli, whether overt or occult ${ }^{1,2}$. Several studies incorporating clinical, radiological, and pathological findings have subsequently evolved but have not led to a consensus on the clinical definition of the disease. Vasakova et al. ${ }^{3}$ proposed two chief groups: acute/inflammatory and chronic/fibrotic HP.

The clinical characteristics of chronic HP can be indistinguishable from those of idiopathic interstitial pneumonia (IIP) particularly in the advanced stages and the histological features on surgical lung biopsy may greatly overlap with those of usual interstitial pneumonia (UIP) and fibrotic nonspecific interstitial pneumonia ${ }^{4}$. Thus, timely identification of chronic 
HP is crucial because evading the inciting antigen is the basis of therapy ${ }^{5,6}$. The presence of peribronchiolar interstitial fibrosis and limited bronchiolo-centric granuloma formation associated with a UIP or nonspecific interstitial pneumonia-like background is indicative of chronic $\mathrm{HP}^{7-10}$.

Several diagnostic tools are essential for making a definitive diagnosis of various interstitial lung diseases (ILDs), including IIPs ${ }^{11}$. Additionally, successive pulmonary function testing is largely used to check the clinical course of the disease and/ or predict the prognosis in patients with ILDs ${ }^{12}$. Conversely, there are various obstacles that have to do with the sensitivity and feasibility of performing repeated assessments; thus, recognition of serum biomarkers to assess and follow the course of ILD would greatly ameliorate contemporary diagnostic methods ${ }^{13-16}$.

Krebs von den Lungen-6 (KL-6) is a circulating high molecular weight mucin-like glycoprotein, also categorized as MUC1, that is expressed primarily on the alveolar type II pneumocytes and the bronchial epithelial cells ${ }^{17,18}$. It is markedly elevated in patients with ILD due to enhanced expression by the regenerating type II pneumocytes and increased permeability after damage of the alveolo-capillary barriers in the lungs affected ${ }^{19}$.

Numerous reports have established that serum KL-6 is a robust prognosticator of consequences of treatment in subjects with acute phases of ILDs and has also been linked to outcomes of survival in subjects with IIP $^{6}$. However, there is limited data regarding the assessment of serum KL-6 levels in chronic HP as compared with other ILDs.

Chronic HP was found to be associated with a further extensive lymphocytic infiltration of the alveoli as compared to interstitial pulmonary fibrosis (IPF)/UIP in a previous report ${ }^{17}$ and Okamato et al. ${ }^{18}$ confirmed a strong correlation between serum KL-6 levels and the proportion of lymphocytes in bronchoalveolar lavage (BAL) and hence suggested that the augmented release of KL-6 into the circulation in chronic HP patients is attributable to impaired bronchial epithelial cells and increased permeability induced by the lymphocytic alveolitis ${ }^{18}$.

The aim of this study was to evaluate the clinical usefulness of serum KL-6 levels as a biomarker for the assessment of disease activity and management of HP.

\section{Materials and Methods}

Patients were recruited from the Department of Chest and Internal Medicine and outpatient clinics, Kasr El Ainy Hospital, Cairo University, during the period between July 2017 and February 2019. The study included 51 patients with HP. Chronic HP patients were retrospectively recruited being previously diagnosed as having chronic HP prior to enrolment, but the acute HP patients were prospectively recruited.
Informed consent was obtained from all participants prior to enrolment. Patients were included in the present study if they were between the age of 20 and 60 years and the diagnosis of HP was established on clinical examination, chest highresolution computed tomography (HRCT), spirometry, arterial blood gas, echocardiography, 6-minute walk test (6MWT), BAL, and transbronchial lung biopsy (TBLB) ${ }^{19}$. Patients with other types of diffuse parenchymal lung diseases; patients with connective tissue diseases or with pulmonary artery pressure $>40 \mathrm{~mm} \mathrm{Hg}$ based on echocardiography; patients with bronchogenic carcinoma or other malignancy and patients with any organ failure were all excluded from the study.

All patients were subjected to detailed history including smoking history, or history of keeping birds, working in fields rich in moldy straw or grains, living in a home with water damage or using a hot tub, sauna, or swimming pool and other possible agents at home or work. The grade of dyspnea was evaluated using the modified Medical Research Council $\operatorname{scale}^{20}$. Laboratory investigations included complete blood count, liver and kidney function tests as well as arterial blood gases analysis.

\section{Assessment of serum KL-6 levels}

Commercially available Bioassay Technology Laboratory enzyme-linked immunosorbent assay kits were used to evaluate the serum levels of human KL-6.

\section{Measurement of serum KL-6}

Three milliliters of whole blood were collected in plain Vacutainer tubes (BD, Franklin Lakes, NJ, USA). Samples were permitted to clot for 10-20 minutes at room temperature. Vacutainer tubes were subjected to centrifugation at 2,000-3,000 rpm for 20 minutes.

\section{Assay procedure}

All reagents, standard solutions and samples were prepared as instructed. The assay was carried out at room temperature. The number of strips required for the assay were determined and inserted into frames. The unused strips were stored at $2-8^{\circ} \mathrm{C}$. A $50 \mu \mathrm{L}$ standard was added to the standard well. Forty microliters of samples were added to sample wells and then $10 \mu \mathrm{L}$ anti-KL-6 antibody were added to sample wells, followed by $50 \mu \mathrm{L}$ streptavidin-horseradish peroxidase which were added to sample wells and standard wells. Proper mixing was performed. The plate was sealed and incubated for an hour at $37^{\circ} \mathrm{C}$, after which it was unsealed and washed 5 times with a buffer. The wells were soaked with $0.35 \mathrm{~mL}$ wash buffer for 30 seconds to 1 minute for each wash. Blotting onto paper towels or another absorbent material was done.

Thereafter, $50 \mu \mathrm{L}$ substrate solution A was inserted into 
each well followed by the addition of $50 \mu \mathrm{L}$ substrate solution B. The plate was resealed and incubated for an average of 10 minutes at $37^{\circ} \mathrm{C}$ in a dark place. Fifty microliters of Stop Solution was then added to each well, and instant change of color from blue to yellow was observed. The optical density (OD value) of each well was determined immediately (within 30 minutes) after addition of the stop solution, using a microplate reader adjusted at $450 \mathrm{~nm}$.

\section{Calculation of results}

A standard curve was created by plotting the average OD for each standard on the vertical (y) axis against the concentration on the horizontal $(\mathrm{x})$ axis and the best fit curve was drawn. The calculations were best accomplished by using computer-based curve-fitting software and the best fit line was determined using regression analysis. The concentration read from the standard curve was multiplied by the dilution factor, when the standard had been diluted.

\section{Chest HRCT}

Scans were obtained at the end of inspiration and in a supine position from the lung apices to the bases and findings were interpreted by a pulmonologist (an expertise in the field).

HRCT patterns distinctive for acute HP included a centrilobular diffuse micronodular pattern, ground-glass opacification, and mosaic attenuation predominantly in upper and middle lobes ${ }^{21}$.

The HRCT findings specific to chronic HP included fibrosis, namely reticulation, architectural distortion, and traction bronchiectasis with or without honeycomb change. The fibrosis may be patchy, peri-bronchovascular, or subpleural (mimicking UIP), and may occur in whichever zonal distribution ${ }^{22}$.

The studied population was scanned for the presence or absence of parenchymal fibrosis. Parenchymal fibrosis was defined as the presence of irregular linear opacities, traction bronchiectasis, or honeycombing ${ }^{23}$. HRCT scans were furthermore evaluated for the presence or absence of ground-glass opacification, centrilobular nodules, and mosaic attenuation.

\section{Pulmonary function test in the form of spirometry}

Spirometry parameters included: forced vital capacity percentage (FVC\%), forced expiratory volume in 1 second $\left(\mathrm{FEV}_{1}\right)$, $\mathrm{FEV}_{1} / \mathrm{FVC}$, and maximal mid-expiratory flow (American Thoracic Society [ATS], 2017). Interpretation of spirometry was done according to the ATS $1994^{24}$.

\section{1) Six-minute walk test}

The 6MWT was performed in all patients using the methodology specified by the ATS $(2002)^{25}$. The patients were instructed to walk as distant as possible for 6 minutes. The
6MWT was carried out in a flat, $30 \mathrm{~m}$ long, covered corridor which was marked at every meter point. Recordings of heart rate and oxygen saturation were documented at the beginning and at the end of the 6MWT. When the test was completed, the elapsed distance was calculated.

\section{2) Bronchoalveolar lavage}

BAL was performed on all patients after informed consent was attained. Analysis of BAL cell counts, culture, and cytology was done. Review radiographs were performed prior to the procedure to define the best site of alveolar lavage. The bronchoscope, collection trap, and tubing were arranged Supplementary oxygen and monitoring apparatus were also prepared and topical sedation with lidocaine $2 \%$ was applied.

The tip of the bronchoscope was advanced to the wedge in a desired subsegmental bronchus. Twenty microliters of saline with a syringe was infused and observed at the distal tip. Gentle suction (50-80 mm Hg) was applied while maintaining wedge position and the lavage specimen was collected in the collection trap. This was repeated for about 5 times. BAL specimens were promptly handled and the required tests were performed.

\section{Transbronchial lung biopsy}

A thorough endobronchial inspection of all segments of both lungs was conducted to exclude significant endobronchial abnormalities. A meticulous airway examination was done prior to the TBLB because bleeding after lung biopsy would interfere with appropriate airway examination. Appropriate control of cough with topical application of lidocaine was essential for ideal biopsy procedure and to decrease the risk of pneumothorax. The choice of biopsy site depended on radiological findings. The biopsy was performed from the dependent parts of the lungs; right or left lower lobes as was favored. In an event of bleeding, the blood is at least confined to this area before spilling into the other lobes.

\section{Technique}

As soon as the biopsy site was selected, the bronchoscope was advanced onto the wedged position by biopsy forceps ${ }^{26}$.

Chest radiographs were performed within 6 hours of transbronchial biopsy in patients developing chest pain or inexplicable hypoxia to exclude pneumothorax. The patient was instructed to tell the staff if they experienced chest pain, hemoptysis, or dyspnea ${ }^{26}$.

\section{Statistical methods}

Data was coded and inserted using an SPSS version 25 (IBM Corp., Armonk, NY, USA). Data was reviewed using mean, standard deviation, median, minimum and maximum for 
quantitative variables and frequencies (number of cases) and relative frequencies (percentages) for categorical variables. Comparisons between groups were done using analysis of variance (ANOVA) with multiple comparisons post-hoc test or unpaired $t$ test in normally distributed quantitative variables while non-parametric Kruskal-Wallis test and MannWhitney test were applied for non-normally distributed quantitative variables ${ }^{27}$. For comparing categorical data, chi-square test was performed. Fisher exact test was used alternatively when the expected frequency was less than $5^{28}$. Correlations between quantitative variables were done using Spearman correlation coefficient ${ }^{29} . \mathrm{p}<0.05$ was considered statistically significant.

\section{Results}

\section{Patient characteristics}

The demographic data of the HP patients in the present study are shown in Table 1. There was a female preponderance among HP patients (84.2\%).

According to the duration of symptoms patients were classified as acute (duration of symptoms $<6$ months) $(8 / 51$ patients, 15.7\%) and chronic (if duration of symptoms $>6$ months) (43/51 patients, $84.3 \%$ ). The symptoms considered were shortness of breath including exertional dyspnea and dry cough. In acute cases, there were additional symptoms considered such as fever and malaise; however, they were infrequently observed in our patients. Only the chronic HP

Table 1. Demographic characteristics of the HP patients

\begin{tabular}{|lc|}
\hline & No. $(\mathbf{\%})(\mathbf{n}=\mathbf{5 1})$ \\
\hline Age $($ mean \pm SD), yr & $39.84 \pm 11.65$ \\
\hline Male & $9(17.6)$ \\
\hline Female & $42(82.4)$ \\
\hline Smoker & $4(7.8)$ \\
\hline Yes & $47(92.2)$ \\
\hline No & \\
\hline Residency & $22(43.1)$ \\
\hline Urban & $29(56.9)$ \\
\hline Rural & \\
\hline Exposure & $35(68.6)$ \\
\hline Birds & $10(19.6)$ \\
\hline Cryptogenic & $6(11.8)$ \\
\hline Others &
\end{tabular}

HP: hypersensitivity pneumonitis; SD: standard deviation. patients $(43 / 51)$ were already on treatment at the time of enrolment but the newly diagnosed acute HP who were enrolled had the KL-6 levels measured before initiation of therapy. The duration of steroid therapy ranged between 16 and 26 months (Table 2) and the dose of steroids ranged between 10 and 20 $\mathrm{mg} /$ day prednisone in the fibrotic group and $20-40 \mathrm{mg} /$ day in the non-fibrotic group. Only 15 patients were on dual therapy of steroids and azathioprine and the dose was $50 \mathrm{mg}$ twice a day or three times a day.

Among a total of 51 HP patients: $35(68.6 \%)$ had a history of bird exposure, six (11.8\%) had a history of exposure to different antigens and in $19.6 \%$ of patients, the inciting antigen could not be detected (cryptogenic). Exposure history included exposure to paints, detergents, plastic (plastic manufacturing), cotton and wool industry and one patient worked as a hairdresser. There was a significant difference between patients and controls regarding the different exposure history $(\mathrm{p}<0.001)$.

\section{Radiological findings on HRCT}

Patients were divided into two groups: the fibrotic (52.9\%) and non-fibrotic (47.1\%) based on the presence or absence of parenchymal fibrosis on HRCT chest. The clinical characteristics of the two groups are demonstrated in Table 2.

The radiologic features of the studied patients are shown in Table 2. All patients in the fibrotic group (100\%) showed irregular linear opacities; however, traction bronchiectasis and honeycombing were observed in $77.8 \%$ and $11.1 \%$, respectively. The HRCT findings of ground-glass opacification, centrilobular nodules, and mosaic attenuation were observed in both groups but the differences were not significant.

\section{Comparison of pulmonary function tests among fibrotic and non-fibrotic HP patients}

When evaluating pulmonary function tests and oxygen requirement among HP patients, FVC (\% of predicted) was found to be significantly lower in the fibrotic group as compared to non-fibrotic group $(52.48 \% \pm 11.30 \%$ and $69.17 \% \pm 12.88 \%$, respectively; $\mathrm{p}<0.001$ ), however, $\mathrm{FEV}_{1} /$ FVC ratio was almost comparable between the two groups (84.22 \pm 8.20 and $82.21 \pm 11.13$, respectively; $\mathrm{p}=0.462$ ) (Table 2 ). The fibrotic group exhibited significantly lower partial pressure of arterial oxygen $(\mathrm{mm} \mathrm{Hg})$ and saturation of arterial oxygen $(\%)$ as compared to the non-fibrotic group $(\mathrm{p}=0.009$ and $\mathrm{p}=0.025$, respectively). Only 13 out of a total of $51 \mathrm{HP}$ patients (25.5\%) required oxygen therapy and $74.5 \%$ were non-oxygen dependent. 
Table 2. Characteristics and radiological HRCT features of HP patients stratified into fibrotic and non-fibrotic groups according to HRCT

\begin{tabular}{|c|c|c|c|c|c|}
\hline \multirow{2}{*}{ Characteristic } & \multicolumn{2}{|c|}{ Fibrotic HP $(n=27 / 51)$} & \multicolumn{2}{|c|}{ Non-fibrotic HP $(n=24 / 51)$} & \multirow{2}{*}{ p-value } \\
\hline & Mean \pm SD & Median & Mean \pm SD & Median & \\
\hline Age, yr & $40.56 \pm 10.51$ & 39 & $39.04 \pm 12.99$ & 35 & 0.648 \\
\hline Dyspnea score & $3.15 \pm 0.86$ & 3 & $2.67 \pm 0.87$ & 3 & 0.053 \\
\hline $\mathrm{PaO}_{2}, \mathrm{~mm} \mathrm{Hg}$ & $62.44 \pm 14.88$ & 65 & $73.88 \pm 15.04$ & 80 & 0.009 \\
\hline 6MWD, m & $279.07 \pm 120.54$ & 280 & $322.92 \pm 119.45$ & 333 & 0.060 \\
\hline Pre-6MWT $\mathrm{SO}_{2}, \%$ & $89.22 \pm 7.71$ & 90 & $93.96 \pm 4.18$ & 95 & 0.010 \\
\hline Post-6MWT SO,$\%$ & $74.15 \pm 10.82$ & 79 & $83.67 \pm 10.89$ & 88 & 0.003 \\
\hline FVC (\% of predicted) & $52.48 \pm 11.30$ & 52 & $69.17 \pm 12.88$ & 71 & $<0.001$ \\
\hline Total dose of steroids, mg/day & $17.6 \pm 10.5$ & 20 & $20.4 \pm 16.4$ & 20 & 0.230 \\
\hline \multicolumn{6}{|l|}{ HRCT findings, n (\%) } \\
\hline GGO & \multicolumn{2}{|c|}{$26(96.3)$} & \multicolumn{2}{|c|}{$23(95.8)$} & $>0.990$ \\
\hline Nodules & 12 & & 11 & & 0.921 \\
\hline Mosaic & 17 & & 12 & & 0.351 \\
\hline Linear reticulations & 27 & & & & $<0.001$ \\
\hline Traction bronchiectasis & 21 & & & & $<0.001$ \\
\hline Honeycombing & & & & & $<0.001$ \\
\hline
\end{tabular}

$\mathrm{p}<0.001$ is considered highly significant; $\mathrm{p}<0.05$ is considered significant.

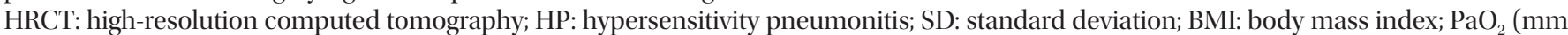
$\mathrm{Hg}$ ): partial pressure of arterial oxygen; $\mathrm{SaO}_{2}$ (\%): saturation of arterial oxygen; 6MWD: 6-minute walk distance; 6MWT: 6-minute walk test; FVC: forced vital capacity; FEV : forced expiratory volume in 1 second; KL-6: Krebs von Lungen-6; GGO: ground-glass opacities.

\section{Evaluation of serum KL-6 levels in HP patients and the effect of therapy}

The median serum KL-6 levels in fibrotic HP group (1,200 $\mathrm{IU} / \mathrm{mL})$ and non-fibrotic group $(1,900 \mathrm{IU} / \mathrm{mL})$ were significantly higher than the control group (140 IU $/ \mathrm{mL}$ ) with a $\mathrm{p}<0.001$. Post-hoc analysis using the Kruskal-Wallis test revealed a significant difference of serum KL-6 levels between fibrotic and non-fibrotic HP with a $\mathrm{p}=0.02$. A significant association was also found between duration of symptoms and serum KL-6 levels with higher values being observed among those patients with duration of symptoms of less than 6 months (median, 2,050 IU/mL) as compared to HP patients with symptoms for more than 6 months (median, 1,300 IU/ $\mathrm{mL}$ ) with a p-value of 0.012 (Table 3 ).

Patients on immunosuppressive therapy showed significantly lower median KL-6 levels as compared to those not on treatment $(1,200 \mathrm{IU} / \mathrm{mL}$ and $1,900 \mathrm{IU} / \mathrm{mL}$ respectively; $\mathrm{p}<0.001$ ) (Table 3). KL-6 levels did not differ significantly be- tween patients on steroids-only regimen and those on dual therapy of steroids and azathioprine (Table 3). However, there was a significant inverse correlation between serum KL-6 levels and the dose and the duration of steroid therapy $(\mathrm{r}=-0.334$, $\mathrm{p}=0.017$ and $\mathrm{r}=-0.442, \mathrm{p}=0.001$, respectively).

When comparing serum KL-6 levels among HP patients with different history of exposure, the mean KL-6 levels were found to be the highest among patients with a history of bird exposure $(1,644.43 \pm 835.71 \mathrm{IU} / \mathrm{mL})$. But the differences in serum KL-6 levels between the causative antigens did not, however, reach statistical significance $(\mathrm{p}=0.515)$ (Table 3 ).

Correlation of serum KL-6 levels with various clinical parameters among HP patients was performed. A significant inverse correlation between serum KL-6 levels and the body mass index (BMI) was only observed $(\mathrm{r}=-0.398, \mathrm{p}=0.004)$; however, KL-6 levels did not show significant correlations with $\mathrm{FVC} \%$, the 6-minute walk distance or other parameters.

Figure 1 represents receiver operating characteristic (ROC) analysis conducted to investigate the ability of KL-6 to dis- 
Table 3. Comparison between KL-6 levels and different clinical parameters

\begin{tabular}{|lcc|}
\hline \multicolumn{1}{|c|}{ Parameter } & $\begin{array}{c}\text { Median KL-6 levels } \\
\text { (IU/L) }\end{array}$ & p-value \\
\hline Exposure & & \\
Birds & 1,500 & 0.515 \\
\hline Others* & 1,200 & \\
\hline Cryptogenic & 1,200 & \\
\hline Smoking & & 0.800 \\
\hline Yes & 1,300 & \\
\hline No & 1,400 & 0.012 \\
\hline Duration of symptoms, mo & & \\
\hline$<6$ & 2,050 & \\
\hline$>6$ & 1,300 & $<0.001$ \\
\hline Therapy & & \\
\hline On treatment & 1,200 & 0.575 \\
\hline No treatment & 1,900 & \\
\hline Type of therapy & & \\
\hline Steroids only & 1,200 & \\
\hline Dual therapy & 1,200 & \\
\hline
\end{tabular}

Dual therapy: steroids and azathioprine.

$\mathrm{p}<0.001$ (highly significant); $\mathrm{p}<0.05$ (significant).

${ }^{*}$ Others: exposure to paints, detergent workers, plastic manufacturing, cotton and wool industry, hairdressing.

KL-6: Krebs von Lungen-6.

criminate between fibrotic HP and non-fibrotic HP patients. It showed an area under the ROC curve (AUC) of 0.807 (95\% confidence interval $[\mathrm{CI}], 0.673-0.941 ; \mathrm{p}<0.001)$ with the best cutoff value of 1,700 with sensitivity $96.3 \%$ but a poor specificity of $62.5 \%$.

\section{Discussion}

In the present study, patients were classified into two groups based on the HRCT chest findings: fibrotic (47.1\%) and nonfibrotic (52.9\%). The fibrotic group exhibited a more restrictive pattern regarding $\mathrm{FEV}_{1}$ and a longer duration of symptoms as compared to the non-fibrotic group. Similarly, a study by Hanak et al. ${ }^{30}$ classified patients into fibrotic and non-fibrotic groups based on HRCT findings of parenchymal fibrosis and demonstrated a longer symptom duration and a greater restrictive lung impairment in fibrotic group as compared to non-fibrotic group ${ }^{30}$.

The median serum KL-6 levels were found to be higher in the non-fibrotic HP group $(1,900 \mathrm{IU} / \mathrm{mL})$ as compared to the fibrotic group $(1,200 \mathrm{IU} / \mathrm{mL})$ in the present study, thus reflecting the response of exposure to various inhaled antigens and

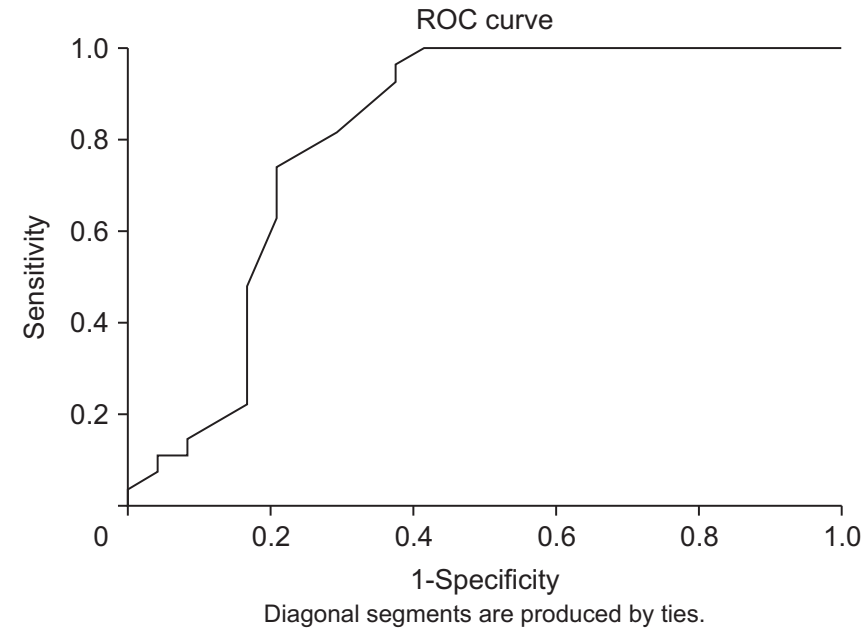

Figure 1. Receiver operating characteristic (ROC) curve showing the ability of Krebs von den Lungen-6 levels to discriminate among fibrotic and non-fibrotic hypersensitivity pneumonitis patients.

the grade of alveolitis. This is in accordance with studies by Ohnishi et al. ${ }^{6}$ and Okamato et al. ${ }^{18}$ that revealed higher serum KL-6 levels in acute non-fibrotic HP when compared to chronic fibrotic HP. The alterations in serum KL-6 concentrations were assumed to be due to alveolar injury and enhanced production of KL-6 by the regenerating type II pneumocytes.

Most of the patients in the present study (68.6\%) had exposure history to birds, $11.8 \%$ had other exposure history mainly occupational, whereas the inciting antigen was unidentifiable in $19.6 \%$ of the patients. Similarly, in a study by Abd El-Kareem et al. ${ }^{31}$ majority of the patients with a diagnosis of HP (68\%) had no history of exposure and $18.6 \%$ of the patients were found to be residing in geographical regions in Egypt that are famous for the exposure to environmental etiologic factors (cane sugar exhaust fumes). Thus, suggesting that some domestic factors, such as indoor (household) molds, dust, along with substantial air pollution, appear to be unrecognizable etiologic factors for HP, so additional investigation is warranted to verify the large number of patients with no exposure history to the frequently recognized antigens.

There was a female preponderance observed among the patients diagnosed with HP in the present study and this could be linked to the causative agent or pathogenesis of the disease itself. A study by Akl et al. ${ }^{32}$ evaluating the demographics of HP in Egypt, reported that out of a total of 118 HP patients, females were ten times more affected than males with a ratio of 10.8:1. That was also in accordance with various studies ${ }^{31,33}$. However, when studying the relationship between the serum KL-6 levels and exposure to different antigens or causative agents for HP, no significant differences were encountered between the type of exposure and median serum levels of KL-6.

Regarding the BMI, it was found to be significantly higher in the fibrotic group compared to the non-fibrotic group. There 
have been conflicting results from previous reports on the association of baseline BMI with outcomes and risk of death in patients with ILD ${ }^{34,35}$. A study by Pugashetti et al. ${ }^{36}$ on patients with ILD with varying etiologies revealed that an annual decline in BMI of $<5 \%$ was associated with a significantly better survival as compared to $\geq 5 \%$ annual decline in BMI and concluded that weight loss was related to an enhanced risk of death in patients with IPF and usual interstitial lung disease, but not in connective tissue disease-ILD or chronic HP. Likewise, a recent study by Comes et al. ${ }^{37}$ also demonstrated a highly significant association between BMI and the 1-year mortality in patients with fibrotic ILD with higher mortality reported in the underweight patients as compared to a lower mortality in the overweight or obese ${ }^{37}$.

ROC analysis was conducted in the present study to investigate the ability of KL-6 to discriminate between fibrotic HP and non-fibrotic HP patients. It showed an AUC of 0.807 (95\% CI, $0.673-0.941 ; \mathrm{p}<0.001$ ) with the best cutoff value of 1,700 with sensitivity $96.3 \%$ but a poor specificity of $62.5 \%$.

In a study by Ohnishi et al. ${ }^{6}$ evaluating the diagnostic utility of KL-6 levels and KL-6 ratio (the variation in the KL-6 concentrations across the patient's clinical course) in discriminating between HP and non-HP, the AUC for the maximum concentration of KL-6 during the patient's clinical course (Kmax) was found to be 0.681 with best cutoff value of 2,532 and a sensitivity of $44.4 \%$ and specificity of $87.2 \%$ (diagnostic accuracy, 79.2\%; $\mathrm{p}=0.017$ ). Moreover, seasonal variations in KL-6 concentrations and KL-6 ratio were found to be significantly more in those with acute HP as compared to chronic HP patients.

There was a statistically significant difference in the median serum KL-6 levels between patients who were already on treatment at the time of enrolment and those without treatment, with higher levels being observed in the latter group. However, the levels did not significantly vary between patients on steroid monotherapy and those on combined therapy (steroids and azathioprine).

In a case report of summer-type HP by Kobayashi et al. ${ }^{38}$ serum KL-6 levels were found to decline gradually with the initiation of steroid therapy. As the dose of steroids was reduced, KL-6 levels decreased below the upper limit of normal and remained persistently low, therefore, emphasizing the clinical utility of KL-6 levels in monitoring of disease activity particularly during steroid withdrawal in patients with interstitial pneumonia ${ }^{38}$. Likewise, Okamato et al. ${ }^{39}$ found that serum KL-6 after treatment was significantly lower than those measured at the start of treatment (median KL-6 levels 1,080 U/ $\mathrm{mL}$ and $1,360 \mathrm{U} / \mathrm{mL}$ respectively; $\mathrm{p}<0.001$ ) and hence implying that estimation of serum KL-6 may be useful in following up the patients.

The precise mechanism of the decline in serum KL-6 levels observed with steroid therapy is still not clearly elucidated. The direct effect of steroids on serum KL-6 is quite improb- able due to lack of glucocorticoid receptor binding site on the promoter area of the MUC1 gene in humans. The role of proinflammatory cytokines namely tumor necrosis factor and interferon- $\gamma$ in enhancing the expression of mucin glycoproteins in human rectal adenocarcinoma cell lines has already been recognized. Accordingly, steroids can decrease serum KL-6 levels via inhibition of the pro-inflammatory cytokines ${ }^{40}$. Another mechanism proposed is decreased permeability of alveolo-capillary barrier with a subsequent reduction in leakage of KL-6 into the circulation.

A few limitations of this study included the small sample size; some of the patients were retrospectively recruited being previously diagnosed as having HP prior to enrolment and hence some of the pathological reports and BAL analysis were lacking. The seasonal variation in serum KL-6 concentrations among the HP patients were also not considered in the present study and so this might have affected the diagnostic accuracy.

The presence of higher KL-6 levels in the non-fibrotic HP group as compared to fibrotic group implies its enhanced production by regenerating pneumocytes in response to alveolar injury. The significant association between serum KL-6 levels and the dose and the duration of steroid therapy emphasized the important role of steroids in stabilization of the disease. Further prospective studies with a larger sample size are warranted to validate the reliability and clinical utility of serum KL-6 as a biomarker for disease severity and prognosis of HP and also to evaluate the relationship between serum KL-6 and cellular pattern in BAL as well as the histological pattern on TBLB in HP patients.

\section{Authors' Contributions}

Conceptualization: Salem AE, Mostafa AI. Methodology: Moussa Ahmed HA, Halim RMA. Formal analysis: Mostafa AI. Data curation: Moussa Ahmed HA. Validation: Abdel Samie RM. Investigation: Bayoumi AI, Halim RMA. Writing - original draft preparation: Mostafa AI. Writing - review and editing: Mostafa AI, Abdel Samie RM. Approval of final manuscript: all authors.

\section{Conflicts of Interest}

No potential conflict of interest relevant to this article was reported.

\section{Funding}

No funding to declare. 


\section{References}

1. Selman M, Pardo A, King TE Jr. Hypersensitivity pneumonitis: insights in diagnosis and pathobiology. Am J Respir Crit Care Med 2012;186:314-24.

2. Inase N, Ohtani Y, Sumi Y, Umino T, Usui Y, Miyake S, et al. A clinical study of hypersensitivity pneumonitis presumably caused by feather duvets. Ann Allergy Asthma Immunol 2006;96:98-104.

3. Vasakova M, Morell F, Walsh S, Leslie K, Raghu G. Hypersensitivity pneumonitis: perspectives in diagnosis and management. Am J Respir Crit Care Med 2017;196:680-9.

4. Akashi T, Takemura T, Ando N, Eishi Y, Kitagawa M, Takizawa T, et al. Histopathologic analysis of sixteen autopsy cases of chronic hypersensitivity pneumonitis and comparison with idiopathic pulmonary fibrosis/usual interstitial pneumonia. Am J Clin Pathol 2009;131:405-15.

5. Ohtani Y, Inase N, Miyake S, Yoshizawa Y, Saiki S. Fatal outcome in chronic bird fancier's lung. Am J Med 2002;112:58890.

6. Ohnishi H, Miyamoto S, Kawase S, Kubota T, Yokoyama A. Seasonal variation of serum KL-6 concentrations is greater in patients with hypersensitivity pneumonitis. BMC Pulm Med 2014;14:129.

7. Churg A, Muller NL, Flint J, Wright JL. Chronic hypersensitivity pneumonitis. Am J Surg Pathol 2006;30:201-8.

8. Hayakawa H, Shirai M, Sato A, Yoshizawa Y, Todate A, Imokawa S, et al. Clinicopathological features of chronic hypersensitivity pneumonitis. Respirology 2002;7:359-64.

9. Ohtani Y, Saiki S, Kitaichi M, Usui Y, Inase N, Costabel U, et al. Chronic bird fancier's lung: histopathological and clinical correlation: an application of the 2002 ATS/ERS consensus classification of the idiopathic interstitial pneumonias. Thorax 2005;60:665-71.

10. Trahan S, Hanak V, Ryu JH, Myers JL. Role of surgical lung biopsy in separating chronic hypersensitivity pneumonia from usual interstitial pneumonia/idiopathic pulmonary fibrosis: analysis of 31 biopsies from 15 patients. Chest 2008;134:12632.

11. Kinnula VL, Ishikawa N, Bergmann U, Ohlmeier S. Proteomic approaches for studying human parenchymal lung diseases. Expert Rev Proteomics 2009;6:619-29.

12. British Thoracic Society Standards of Care Committee. The diagnosis, assessment and treatment of diffuse parenchymal lung disease in adults. Introduction. Thorax 1999;54 Suppl 1:S1-14.

13. Low RB, Giancola MS, King TE Jr, Chapitis J, Vacek P, Davis GS. Serum and bronchoalveolar lavage of N-terminal type III procollagen peptides in idiopathic pulmonary fibrosis. Am Rev Respir Dis 1992;146:701-6.

14. McCormack FX, King TE Jr, Bucher BL, Nielsen L, Mason RJ. Surfactant protein A predicts survival in idiopathic pulmonary fibrosis. Am J Respir Crit Care Med 1995;152:751-9.
15. Yokoyama A, Kohno N, Hirasawa Y, Kondo K, Abe M, Inoue Y, et al. Evaluation of soluble IL-6 receptor concentration in serum and epithelial lining fluid from patients with interstitial lung diseases. Clin Exp Immunol 1995;100:325-9.

16. Jack CI, Jackson MJ, Johnston ID, Hind CR. Serum indicators of free radical activity in idiopathic pulmonary fibrosis. Am J Respir Crit Care Med 1996;153:1918-23.

17. Takemura T, Akashi T, Kamiya H, Ikushima S, Ando T, Oritsu M, et al. Pathological differentiation of chronic hypersensitivity pneumonitis from idiopathic pulmonary fibrosis/usual interstitial pneumonia. Histopathology 2012;61:1026-35.

18. Okamoto T, Tsutsui T, Suhara K, Furusawa H, Miyazaki Y, Inase N. Seasonal variation of serum KL-6 and SP-D levels in bird-related hypersensitivity pneumonitis. Sarcoidosis Vasc Diffuse Lung Dis 2015;31:364-7.

19. Patel RA, Sellami D, Gotway MB, Golden JA, Webb WR. Hypersensitivity pneumonitis: patterns on high-resolution CT. J Comput Assist Tomogr 2000;24:965-70.

20. Fletcher CM. The clinical diagnosis of pulmonary emphysema; an experimental study. Proc R Soc Med 1952;45:57784.

21. Johannson KA, Elicker BM, Vittinghoff E, Assayag D, de Boer $\mathrm{K}$, Golden JA, et al. A diagnostic model for chronic hypersensitivity pneumonitis. Thorax 2016;71:951-4.

22. Silva CI, Muller NL, Lynch DA, Curran-Everett D, Brown KK, Lee KS, et al. Chronic hypersensitivity pneumonitis: differentiation from idiopathic pulmonary fibrosis and nonspecific interstitial pneumonia by using thin-section CT. Radiology 2008;246:288-97.

23. Glazer CS, Rose CS, Lynch DA. Clinical and radiologic manifestations of hypersensitivity pneumonitis. J Thorac Imaging 2002;17:261-72.

24. Standardization of spirometry, 1994 update. American Thoracic Society. Am J Respir Crit Care Med 1995;152:1107-36.

25. American Thoracic Society; European Respiratory Society. American Thoracic Society/European Respiratory Society International Multidisciplinary Consensus Classification of the Idiopathic Interstitial Pneumonias. This joint statement of the American Thoracic Society (ATS), and the European Respiratory Society (ERS) was adopted by the ATS board of directors, June 2001 and by the ERS Executive Committee, June 2001. Am J Respir Crit Care Med 2002;165:277-304.

26. Izbicki G, Shitrit D, Yarmolovsky A, Bendayan D, Miller G, Fink $\mathrm{G}$, et al. Is routine chest radiography after transbronchial biopsy necessary?: a prospective study of 350 cases. Chest 2006;129:1561-4.

27. Chan YH. Biostatistics 102: quantitative data: parametric and non-parametric tests. Singapore Med J 2003;44:391-6.

28. Chan YH. Biostatistics 103: qualitative data: tests of independence. Singapore Med J 2003;44:498-503.

29. Chan YH. Biostatistics 104: correlational analysis. Singapore Med J 2003;44:614-9.

30. Hanak V, Golbin JM, Hartman TE, Ryu JH. High-resolution CT 
findings of parenchymal fibrosis correlate with prognosis in hypersensitivity pneumonitis. Chest 2008;134:133-8.

31. Abd El-Kareem D, Akl YM, Nakhla GA, Elhindawi AA, Eltorky MA. Clinico-pathologic presentation of hypersensitivity pneumonitis in Egyptian patients: a multidisciplinary study. Multidiscip Respir Med 2017;12:10.

32. Akl Y, Soliman YM, El-Korashy RI, El-Hennawi YH. Demographic study of hypersensitivity pneumonitis in Egypt: a single center experience. Am J Respir Crit Care Med 2017;195: A1566.

33. Adegunsoye A, Oldham JM, Demchuk C, Montner S, Vij R, Strek ME. Predictors of survival in coexistent hypersensitivity pneumonitis with autoimmune features. Respir Med 2016;114:53-60.

34. Alakhras M, Decker PA, Nadrous HF, Collazo-Clavell M, Ryu JH. Body mass index and mortality in patients with idiopathic pulmonary fibrosis. Chest 2007;131:1448-53.

35. Kondoh Y, Taniguchi H, Katsuta T, Kataoka K, Kimura T, Nishiyama O, et al. Risk factors of acute exacerbation of idiopathic pulmonary fibrosis. Sarcoidosis Vasc Diffuse Lung Dis
2010;27:103-10.

36. Pugashetti J, Graham J, Boctor N, Mendez C, Foster E, Juarez $\mathrm{M}$, et al. Weight loss as a predictor of mortality in patients with interstitial lung disease. Eur Respir J 2018;52:1801289.

37. Comes A, Wong AW, Fisher JH, Morisset J, Johannson KA, Fell CD, et al. Association of body mass index and change in weight with mortality in patients with fibrotic interstitial lung disease. Am J Respir Critical Care Med 2020;201:A1096.

38. Kobayashi J, Tsukagoshi M, Hagiwara S, Kitamura S, Hirota N, Saito K. Hypersensitivity pneumonitis monitored with serum KL-6, a marker of interstitial pneumonia. Nihon Kyobu Shikkan Gakkai Zasshi 1996;34:837-42.

39. Okamoto T, Fujii M, Furusawa H, Tsuchiya K, Miyazaki Y, Inase N. The usefulness of KL-6 and SP-D for the diagnosis and management of chronic hypersensitivity pneumonitis. Respir Med 2015;109:1576-81.

40. Inoue Y, Barker E, Daniloff E, Kohno N, Hiwada K, Newman LS. Pulmonary epithelial cell injury and alveolar-capillary permeability in berylliosis. Am J Respir Crit Care Med 1997; 156:109-15. 